\title{
Actitudes en Centros Educativos respecto a la Educación para la Ciudadanía Global
}

\section{Attitudes in Schools regarding Education for Global Citizenship}

\author{
Ana Cristina Blasco-Serrano \\ Belén Dieste * \\ Teresa Coma
}

Universidad de Zaragoza, España

\begin{abstract}
En el siglo XXI, la educación tiene el reto de ayudar al individuo a comprender el mundo y al otro, para comprenderse mejor a sí mismo. En este marco, la Educación para la Ciudadanía Global (ECG) se presenta como un eje para la transformación social. En este trabajo se analizan enfoques educativos, a partir de las prácticas de ECG desarrolladas en distintos centros educativos. Se muestra la investigación realizada con equipos docentes de Educación Primaria y Secundaria de centros del ámbito rural de la provincia de Zaragoza. El diseño metodológico es un estudio descriptivo no experimental, tomando como muestra a 77 centros educativos, a través de un cuestionario autoadministrado. El clúster bietápico entre la existencia de acciones ECG coordinadas, los motivos por los que se desarrollan y los aspectos de mejora necesarios, ha puesto de relieve tres tipos de actitudes existentes en los centros: pasivas, receptivas y proactivas, que cobran especial relevancia al conectarlos con el resto de las variables. En la discusión se señala la importancia de sensibilizar a toda la comunidad educativa para que se creen sinergias que desarrollen nuevas propuestas e iniciativas a través de proyectos planificados, participativos y a largo plazo. De este modo, la actitud de los docentes es un pilar básico, pero el apoyo institucional será imprescindible para dar estabilidad a estas propuestas.
\end{abstract}

Descriptores: Educación para la ciudadanía global; Escuela rural; Profesorado; Comunidad educativa; Actitudes.

In XXI century, education has the challenge of helping to people to understand the world and the other, in order to better understand oneself. In this framework, Education for Global Citizenship (ECG) is presented as an axis for social transformation. In this work, the experience of teaching teams in ECG practices is analyzed. The research carried out with educational teams of Primary and Secondary schools of the rural area of the province of Zaragoza is shown. The methodological design is a non-experimental descriptive study, taking as sample 77 schools, through a self-administered questionnaire. The two-stage cluster between the existence of coordinated ECG actions, the reasons for which they are developed, and the necessary aspects of improvement has highlighted three types of attitudes existing in the schools: passive, receptive and proactive, which become relevant when connected to the rest of the variables. The discussion points out the importance of sensitizing the entire educational community so that new proposals and initiatives are developed through planned, participatory and long-term projects. In this way, the attitude of teachers is a basic pillar, but institutional support will be essential to give stability to these proposals.

Keywords: Education for global citizenship; Rural school; Teachers; Educative community; Attitudes.

*Contacto: bdieste@unizar.es

Recibido: $\quad 19$ de marzo 2019

ISSN: $1696-4713$

$1^{\text {a }}$ Evaluación: 25 de abril 2019

www.rinace.net/reice/

$2^{\text {a }}$ Evaluación: 10 de mayo 2019

revistas.uam.es/reice

Aceptado: $\quad 24$ de mayo 2019 


\section{Revisión de la literatura}

La Declaración de Incheon (UNESCO, 2015a), en el Marco de Acción de Educación 2030, pone de relieve la importancia de una educación transformadora. Bajo esta visión humanista y universal se promueve el desarrollo y la consecución del Objetivo de Desarrollo Sostenible-4 (ODS-4) de la Agenda 2030: "Garantizar una educación inclusiva y equitativa de calidad y promover oportunidades de aprendizaje permanente para todos".

Desde esta perspectiva, la educación es un medio para conseguir el cambio social, y por tanto la mirada del docente se proyecta hacia el horizonte (Bowers, 2004; Wang y Hoffman, 2016), especialmente cuando se habla de hacer las cosas de otra manera y de construir el proceso educativo entre todos. De este modo, tal y como señala la UNESCO (2015b), todas las acciones educativas deberían estar en conexión con la meta oDs 4.7:

Asegurar que todos los alumnos adquieran los conocimientos teóricos y prácticos
necesarios para promover el desarrollo sostenible, entre otras cosas mediante la
educación para el desarrollo sostenible y los estilos de vida sostenibles, los derechos
humanos, la igualdad de género, la promoción de una cultura de paz y no violencia,
la ciudadanía mundial y la valoración de la diversidad cultural y la contribución de
la cultura al desarrollo sostenible. (UNESCO, $2015 \mathrm{~b}, \mathrm{p} .21$ )

La educación, como ya indicaba Delors y otros (1996, p. 31), deberá “ayudar a comprender el mundo y a comprender al otro, para así comprenderse mejor a sí mismo" y todo ello, desde la inclusión y el respeto de los derechos individuales. Una mayor comprensión mutua, intensificar el sentido de la responsabilidad y de la solidaridad, así como aceptar nuestras diferencias culturales son considerados aspectos clave para la ECG, y principios fundamentales que deben orientar la educación del siglo XXI (Celorio, 2013; Engel, Fundalinski y Cannon, 2016).

La Educación para la Ciudadanía Global (ECG) se revela como un medio para evolucionar hacia transformaciones alternativas, en favor de una sociedad más justa, solidaria y equitativa (Peña, Delgado y Valverde, 2014; Pigozzi, 2006). Para ello, se hace necesario incorporar en los procesos educativos una visión del mundo global y preparar a las personas para actuar con la responsabilidad y el compromiso que implica la ciudadanía global (Boni, 2011).

En este escenario, la integración de la ECG en la educación formal se presenta como una herramienta clave desde la que educar a ciudadanos críticos, cooperativos y solidarios, que promuevan cambios y transformaciones en un mundo cada vez más complejo (BoniAristizabal, Hofmann-Pinilla y Sow-Paino, 2012). Una educación transformadora ha de proporcionar diferentes perspectivas, para que, a partir del pensamiento analítico, crítico y creativo, se puedan generar cambios en las actitudes y el comportamiento de los ciudadanos (Brown, 2018). Esta reconstrucción implica reflexionar y cuestionar el propio sistema y las prácticas educativas para buscar alternativas y reelaborar propuestas (Vigo, Dieste y Thurston, 2016). Los centros educativos deberán crear redes colaborativas de trabajo, esfuerzos conjuntos y proyectos comunitarios donde los profesionales de la educación ejerzan como motor de cambio, examinando el currículo, así como, diseñando y desarrollando propuestas de ECG (Digón et al., 2017; Kopish, 2017).

El cambio en esta dirección será realmente efectivo cuando estas acciones educativas sean globales e institucionales (Brown, 2018; Contreras y Aceituno, 2018). La literatura científica señala que los responsables de las políticas educativas están cada vez más orientados a incluir la ECG en la formación inicial del profesorado (Estellés y Romero, 
2016; Kopish, 2017), en el currículo y en los centros educativos (Goren y Yemini, 2017; Guo, 2014). No obstante, también se insiste en la necesidad de una mayor investigación y reflexión en torno a la teoría y la práctica que permita aclarar el significado y el sentido de la ECG en la educación formal (Boni-Aristizabal, Hofmann-Pinilla y Sow-Paino, 2012) como punto de partida.

En este marco, será necesario analizar cómo trasladar todos estos aspectos a la práctica educativa, ¿cómo hacer que uno mismo sea consciente de su propio pensamiento en conexión con la acción, si no intercambia con otros? ¿cómo sentirse parte de algo si no se percibe cómo se construye?, ¿cómo saber qué es construir con otros? En los centros educativos, desde la teoría y la práctica, es posible desarrollar la ECG desde una consciencia activa, contribuyendo a la mejora de la convivencia social, a la inclusión de los grupos minoritarios, a comprender los diferentes escenarios y contextos culturales y a que las personas tengan las habilidades necesarias para acceder a la información (Cabrera, 2007).

La ECG requiere un aprendizaje incluyente, en el que se aprenda a comprender y percibir a las otras personas, donde se aprenda desde la interdependencia y todo ello con autonomía y responsabilidad. Es decir, "el conjunto de conocimientos, destrezas y actitudes es esencial para que todos los individuos, especialmente aquellos en riesgo de exclusión, puedan participar activamente e integrarse como miembros de la sociedad" (Bolívar, 2010, p. 23). La ECG implica que la persona sea agente de su proceso de aprendizaje con una actitud empática. Para ser un ciudadano de un mundo globalizado, se considera imprescindible la participación en la comunidad ya que, "un ciudadano del mundo es ciertamente de alguna parte, con raíces en lo local” (Amiguinho, 2011, p. 11). Y, por lo tanto, un ciudadano con competencia global es según Kikuchi (2003) una persona que tiene el conocimiento, las habilidades y las actitudes para interactuar de manera efectiva en una sociedad globalmente interdependiente.

La dualidad, local-global, lejos de aparecer como opuesta, pasa a contener las dos caras, perspectivas necesarias de cualquier acto ciudadano, responsable y significativo. Una orientación hacia el "bien común" o búsqueda de un beneficio mayor que el meramente personal (Maiztegui, 2007).

\subsection{La ECG en la escuela rural}

La escuela rural no es ajena al proceso de globalización ni a la necesidad de interdependencia entre las comunidades. Así, desde esta perspectiva en la que se valora lo local y propio, el alumnado aprende a mirarse y reconocerse también en lo global, convirtiéndose lo ajeno en algo propio (Sepúlveda Ruiz y Gallardo Gil, 2011).

En el ámbito geográfico español, esta escuela se enmarca, en muchos casos, en la denominada por Del Molino (2016) como "España vacía”. En el caso de la provincia de Zaragoza, existe un gran centro urbano (Zaragoza) que concentra la mayoría de recursos y servicios, mientras que el resto del territorio se encuentra, en su mayoría, intensamente despoblado.

Estas características geográficas, sociales y económicas configuran una escuela rural con peculiaridades, como las aulas multigrado, la inestabilidad del profesorado o la disgregación geográfica. No obstante, estas características crean a su vez, en muchos casos, espacios educativos de convivencia que no toman únicamente como referencia lo local, sino que consideran la diversidad como un valor para crear una cultura de convivencia (Torres, 2011). Todos estos valores, conforman el reflejo del sentido de 
convivencia de la comunidad rural (Boix, 2011). En este marco, se desarrollan prácticas que unen la escuela con su comunidad, con su cultura y con su identidad, promoviendo así, la sostenibilidad de la escuela y de su entorno. Estas prácticas requieren de la aplicación de metodologías activo-participativas, que originen contextos de aprendizaje significativos y creativos (Vigo y Soriano, 2014).

Trabajar en las aulas a partir de los intereses del alumnado permite desarrollar en los niños y niñas la curiosidad hacia nuevos conocimientos. En consecuencia, se incentiva una búsqueda orientada a saber más, a reflexionar, discutir, y experimentar a partir de una gran diversidad de modalidades de actividades de aprendizaje, en favor de un pensamiento creativo (Boix, 2011). De este modo, desde la escuela rural, se construyen puentes entre el currículo y la comunidad de una forma más natural que en las zonas urbanas (Vigo y Dieste, 2018). Este vínculo entre escuela y comunidad local facilita el trasvase de significados del territorio al currículum y viceversa. No se puede obviar esta ventaja pedagógica que ofrece el contexto rural (Boix, 2011). Esta concepción de la escuela rural se entronca con un compromiso de justicia social y educativa con su entorno, así como de reconstrucción de formas de acción social (Sepúlveda Ruiz y Gallardo Gil, 2011).

\subsection{Papel del profesorado}

En este escenario, el desarrollo personal y profesional de los agentes educativos es clave. Más concretamente, el conocimiento y la sensibilidad de los docentes sobre la Ciudadanía Global será un aspecto básico en el desarrollo de la ECG en los centros educativos (Banks, 2017).

La educación se ha de orientar hacia el dominio de estrategias y habilidades para una visión crítica y comprometida de la realidad, que desarrolle valores e ideales susceptibles de ser superados y transformados (Gimeno Sacristán, 2001). Si no se toma consciencia de que la prioridad es convertir a los estudiantes en sujetos activos de su propio aprendizaje, difícilmente se posibilitarán experiencias que coloquen a los estudiantes en el centro, facilitando que sean agentes activos, con curiosidad y sentido crítico. En este sentido, cuando los docentes articulan propuestas de actuación, que tienen como eje el aprendizaje de una ciudadanía global, revalorizan y enriquecen su función social desde los principios de equidad y justicia social (Rumayor y de las Heras, 2012).

En el caso de las escuelas rurales, estas experiencias surgen, normalmente, en la confluencia de diferentes aspectos. De una parte, de una valoración muy positiva de la escuela por parte de las familias y la comunidad debida, entre otros aspectos, a relaciones de proximidad. De otra parte, por un trabajo escolar de los niños y niñas, del profesorado e incluso de otros actores sociales, que las circunstancias ayudan a promover (Amiguinho, 2011; Gómez-Quintero et al., 2017). No podemos obviar que, en el caso del profesorado de las escuelas rurales, con frecuencia, se encuentran en una situación de enseñanzaaprendizaje para la que, en la mayoría de las ocasiones, no han sido formados y, en consecuencia, actúan repitiendo modelos diseñados principalmente para una escuela urbana, con características muy distintas a las de la escuela rural (Boix, 2011). Y que, además, tal y como explican Boni-Aristizábal, Hofmann-Pinilla y Sow-Paino (2012), "la ED de quinta generación o ECG adolece de mayor trabajo conjunto entre los diferentes actores que la practican" (p. 75).

De este modo, el profesorado se presenta, en todos los escenarios, como el principal agente responsable de educar para una Ciudadanía Global (García y De Alba, 2009; Willemse et 
al., 2015). Actualmente, muchos indicadores muestran que, pese a existir un profesorado conceptualmente proclive a trabajar en esta dirección, factores como la limitación del tiempo, la sobrecarga de trabajo o las restricciones del propio currículum, hace que sean un número limitado de docentes los que lleven a la práctica el enfoque educativo orientado a la Ciudadanía Global (Blasco-Serrano, Coma y Dieste, 2018; Gómez-Quintero et al., 2017).

En este marco, este estudio tiene como finalidad analizar y reflexionar sobre las diferentes actitudes del profesorado de Educación Primaria y Secundaria, de centros educativos del ámbito rural de la Provincia de Zaragoza, respecto a la incorporación en la práctica educativa de la ECG en las dinámicas del centro y en los procesos de enseñanzaaprendizaje.

\section{Método}

Este estudio descriptivo exploratorio tiene como finalidad analizar enfoques educativos, a partir de las prácticas de ECG desarrolladas en distintos centros educativos. Se muestra la investigación realizada con equipos docentes de Educación Primaria y Secundaria de centros del ámbito rural de la provincia de Zaragoza. Se presenta el análisis de los datos obtenidos a través de un cuestionario auto-administrado.

\section{Objetivos}

Los objetivos específicos del presente estudio se concretan en:

- Analizar diferentes actitudes del profesorado de Educación Primaria y Secundaria, respecto a la incorporación en la práctica educativa de la ECG.

- Relacionar las diferentes actitudes del profesorado con relación al sentido que se le atribuye a las actuaciones de ECG.

- Indagar en cómo los centros planifican y con quién se coordinan para llevar a cabo actuaciones de ECG.

\section{Diseño metodológico}

En esta investigación se ha desarrollado un diseño descriptivo, a través de una encuesta social (Briones, 1996; Sabirón, 2006). El estudio forma parte de una investigación más amplia que tiene como objetivo el diagnóstico de la educación para la ciudadanía global en la provincia de Zaragoza.

\section{Población y muestra}

En el estudio han participado los equipos docentes de 77 centros educativos de los 147 existentes en la provincia de Zaragoza sostenidos con fondos públicos; 54 de ellos son de Educación Infantil y Primaria, 5 de Educación Infantil, Primaria y Secundaria y 18 de Educación Secundaria. La muestra representa el 52.4\% de los centros sostenidos con fondos públicos, pertenecientes a 74 localidades diferentes, situadas fuera del área metropolitana de Zaragoza. Como se puede observar en la Figura 1, la mayoría de las localidades tienen menos de 5000 habitantes. 


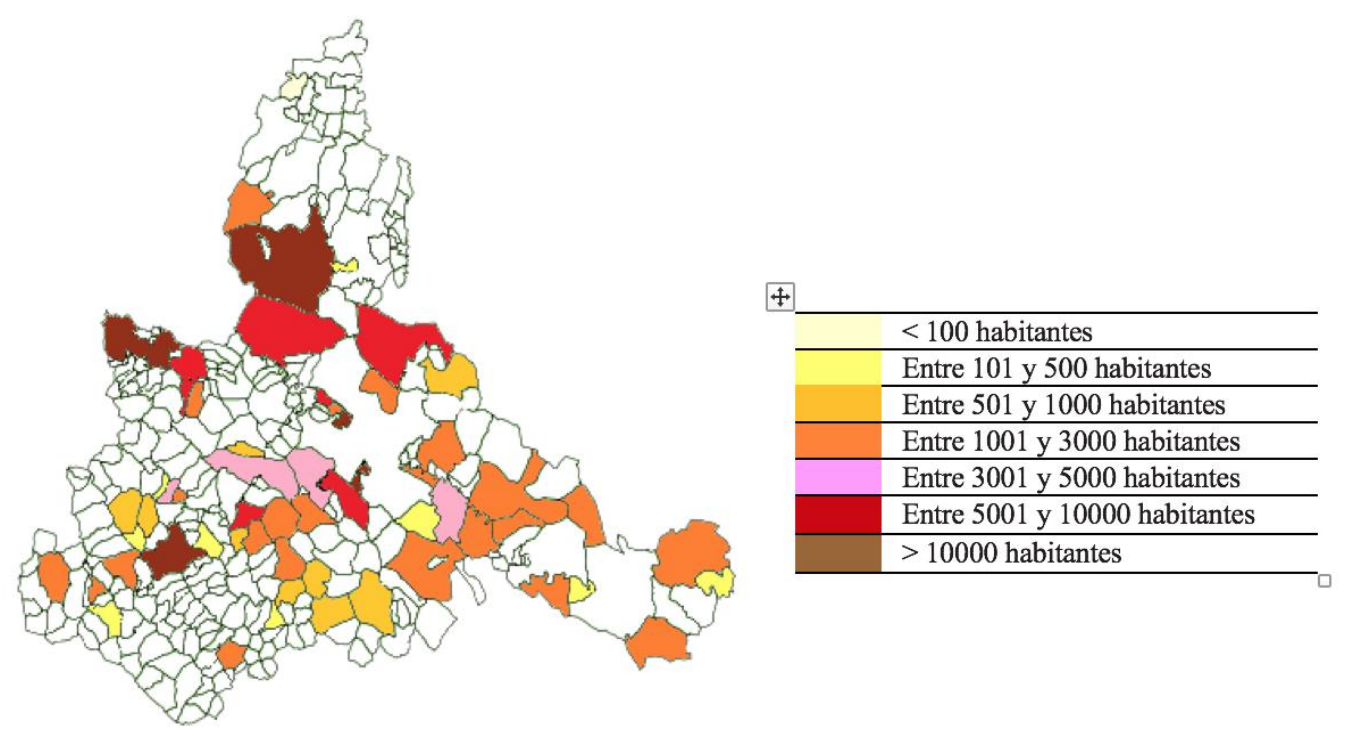

Figura 1. Distribución geográfica y poblacional de las localidades participantes Fuente: Elaboración propia.

\section{Instrumento}

El cuestionario utilizado como método de recogida de información fue validado, en el marco del Proyecto de EuropeAid (OCUVA, 2017), por profesionales de la educación de siete países europeos (España, Bulgaria, Chipre, Grecia, Italia, Malta y Polonia). La coordinación se llevó a cabo por el Observatorio de Cooperación de la Universidad de Valladolid.

Es un cuestionario estructurado de tipo descriptivo transversal auto-administrado (Gómez, Domenech y Carracedo, 2012). En una primera parte, el cuestionario recoge datos para la identificación del centro y del equipo docente, mientras que, en la segunda parte, trece preguntas hacen referencia al desarrollo de la ECG en el centro educativo. Dos de estas preguntas son de respuesta dicotómica (si/no), ocho son de respuesta múltiple de tipo cualitativo, una es cerrada de valor numérico, y tres son preguntas abiertas. El cuestionario está organizado en 5 bloques, en los cuales se incluyen las variables analizadas (cuadro 1).

Recogida y análisis de datos

El cuestionario se envió por correo electrónico a todos los centros de la provincia de Zaragoza en formato Google Forms. En cada centro, participa una persona del equipo docente, con el fin de reflejar la percepción global sobre la ECG en el claustro docente y la realidad educativa de su centro.

Se realizó un análisis temático (Braun y Clarke, 2006) de las dos preguntas abiertas referentes a las variables motivación del profesorado y propuestas de mejora. En el análisis temático, en un primer lugar, surgieron unas categorías iniciales al leer con detalle los datos, buscando estructuras y significados. La categorización ha sido de tipo inductivo, emergiendo los códigos durante el proceso sistemático y reiterativo del análisis de la información. De esta manera, las categorías finalmente definidas (figura 2), delimitan y describen las situaciones de los centros educativos en relación a la ECG. 
Cuadro 1. Estructura del cuestionario

\begin{tabular}{ll}
\hline \multicolumn{1}{c}{ BLOQUES } & \multicolumn{1}{c}{ VARIABLES } \\
\hline \multirow{2}{*}{ Acciones } & $\begin{array}{l}\text { Realización de acciones de ECG en el centro escolar. } \\
\text { Coordinación para la realización de acciones en el propio centro o con otros } \\
\text { agentes para trabajar la ECG. } \\
\text { Tipo de acciones para la ECG que se llevan a cabo. }\end{array}$ \\
\hline Implicación & $\begin{array}{l}\text { Agentes que toman la iniciativa ante la ECG. } \\
\text { Origen de los materiales sobre la ECG. } \\
\text { Número de profesores implicados en las acciones de ECG. }\end{array}$ \\
\hline Contenidos & Contenidos sobre ECG en Educación Primaria y en Educación Secundaria. \\
\hline Sentido & $\begin{array}{l}\text { Motivación del profesorado para implicarse en la ECG. } \\
\text { Consideración sobre la necesidad de la ECG. }\end{array}$ \\
\hline $\begin{array}{l}\text { Aspectos de } \\
\text { mejora }\end{array}$ & \begin{tabular}{l} 
Propuestas de mejora respecto a la ECG. \\
\hline Fuente: Elaboración propia, a partir del cuestionario del Proyecto de EuropeAid (OCUVA, 2017).
\end{tabular}
\end{tabular}

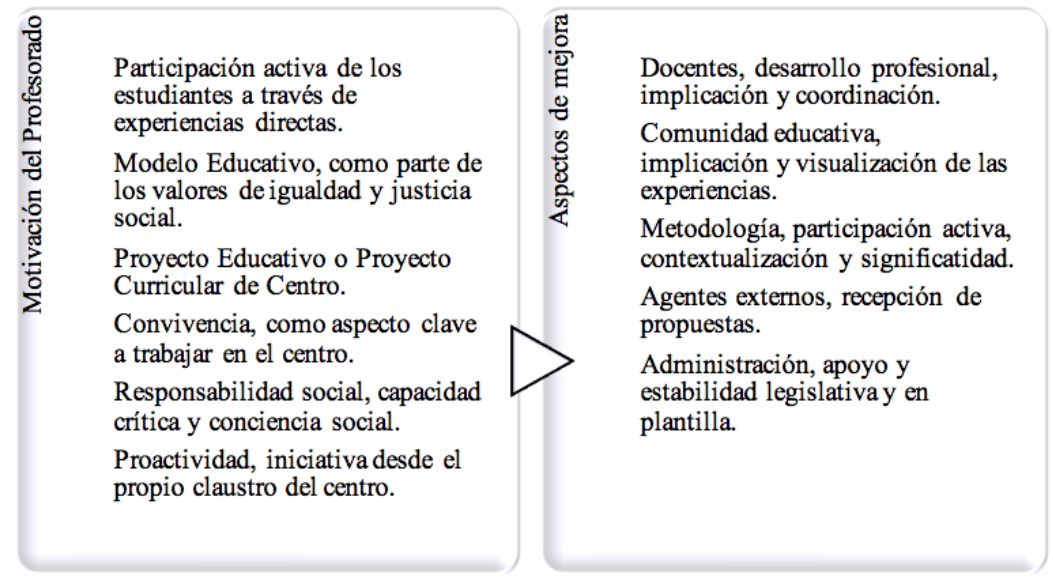

Figura 2. Categorización motivación del profesorado y aspectos de mejora Fuente: Elaboración propia.

Además, se ha realizado un estudio exploratorio, mediante el análisis estadístico de conglomerados o clúster bietápico. Esta herramienta ha permitido descubrir agrupaciones para clasificar los perfiles de equipos docentes según las diferentes actitudes con relación a la ECG, así como establecer similitudes o diferencias entre los mismos (Rubio-Hurtado y Vilà-Baños, 2017; Soriano y Monsalve, 2004).

Tras este análisis exploratorio de todas las variables, los agrupamientos obtenidos en el clúster bietápico (que incluye cuatro variables), se relacionaron con el resto de las variables del estudio. Todo ello, a través del programa estadístico IBM SPSS Statistics 22.

\section{Resultados}

Las cuatro variables que han permitido clasificar los perfiles de equipos docentes han sido:

- Realización de acciones de ECG en el centro escolar.

- Coordinación para la realización de acciones en el propio centro o con otros agentes para trabajar la ECG.

- Motivación del profesorado para implicarse en la ECG. 
- Propuestas de mejora respecto a la ECG.

Esta clasificación, tal y como se muestra en la figura 3, diferencia tres tipos de equipos de centro denominados: pasivos (grupo 1), receptivos (grupo 2) y proactivos (grupo 3).

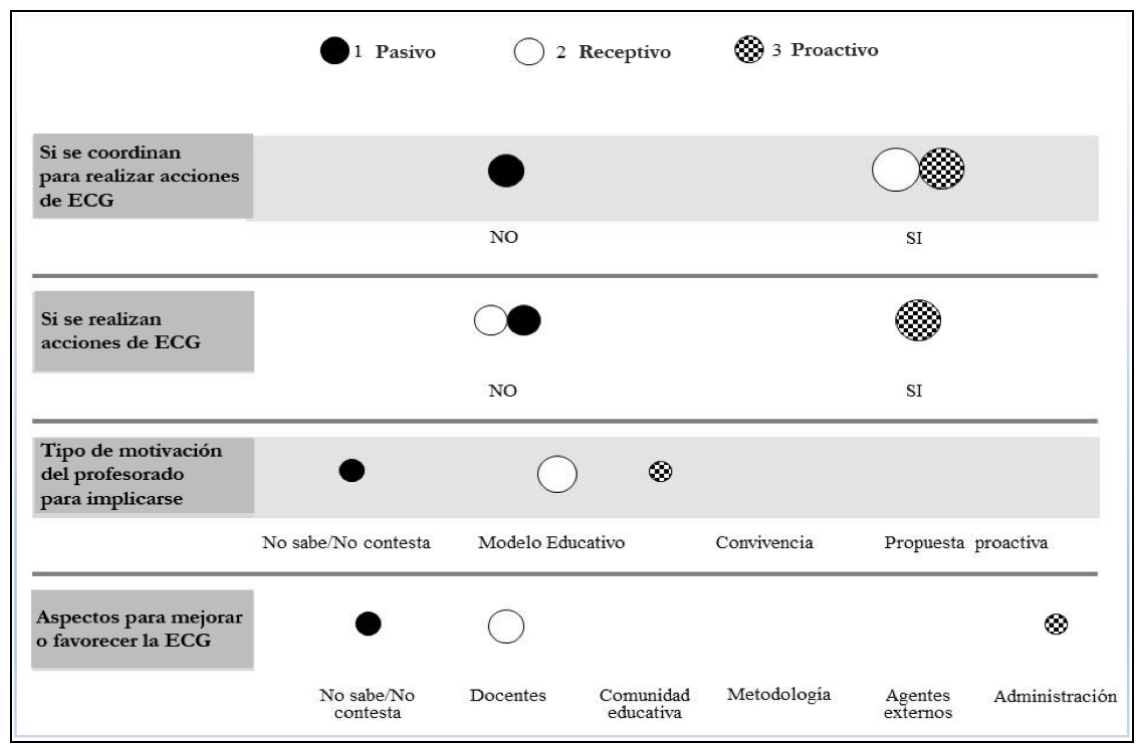

Figura 3. Tipos de perfiles de equipos docentes en relación a la ECG

Fuente: Elaboración propia.

El 22,10\% (17 equipos docentes) forman parte de la categoría de pasivos, puesto que no se recogen acciones sobre ECG en el Proyecto Educativo de Centro (PEC), y, por tanto, tampoco hay acciones coordinadas, además no expresan sus motivos para realizarlas y tampoco mejoras de futuro.

Los receptivos representan un 42,80\% (33 centros), y se caracterizan por no incluir acciones sobre ECG en el PEC por iniciativa propia, pero sí se coordinan para realizar acciones. El motivo principal que señalan para participar es que su modelo educativo prioriza el desarrollo de valores y actitudes, aunque se observan diferentes matices. Mientras unos priorizan la formación integral, otros entienden que son parte del currículo, en otros casos entienden que son una apuesta del PEC, y otro grupo considera que su prioridad es la educación en valores.

Los aspectos de mejora que plantean corresponden al ámbito de los docentes, ya que se considera prioritario un mayor desarrollo profesional, implicación y coordinación del profesorado.

Los proactivos, son un $35,10 \%$ (27 centros), desarrollan acciones de ECG de forma coordinada. El principal motivo para participar, al igual que los receptivos, es su idea de modelo educativo. Pero se incide más en la convivencia (respeto a la diversidad) como aspecto práctico a desarrollar en el centro. Estos centros resaltan como mejora los aspectos relativos a la Administración (estabilidad en la plantilla, normativa y mayor apoyo de las instituciones públicas).

No se han observado diferencias significativas por tipología de centro en relación al nivel educativo del centro, tiempo y distancia de los centros rurales respecto a la capital, número de habitantes de la localidad, número de docentes o de estudiantes, tipo de contenidos 
abordados de ECG, ni en la forma de desarrollar las actividades en el centro (específica en una asignatura o materia transversal en diferentes asignaturas o materias y de forma específica y transversal).

Sin embargo, se puede observar que la mayoría de los equipos, son receptivos en igual porcentaje en Primaria que en Secundaria $(45,80 \%)$. Por otra parte, en Educación Primaria hay más equipos de tipo proactivo (38,98\% frente a un 20,83\% de Educación Secundaria); y en Educación Secundaria priman los de tipo pasivo (33,33\% frente a un 15,25\% en E. Primaria). También se observa una tendencia a que los equipos de los centros más pequeños sean más proactivos, ya que realizan más acciones y actividades en ECG.

Destacan cinco variables que muestran diferencias significativas entre los tres tipos de equipos docentes: grado de necesidad de enseñar ECG en horario lectivo, con qué agentes se coordinan para realizar acciones de ECG, tipos de acciones educativas desarrolladas de ECG, qué agentes educativos toman la iniciativa para desarrollar acciones de ECG y cuál es la fuente de los materiales empleados en ECG.

En cuanto al grado de necesidad de enseñar ECG en horario lectivo, se observan diferencias significativas, tal y como se muestra en la figura $4 \mathrm{y}$ en el cuadro 2.

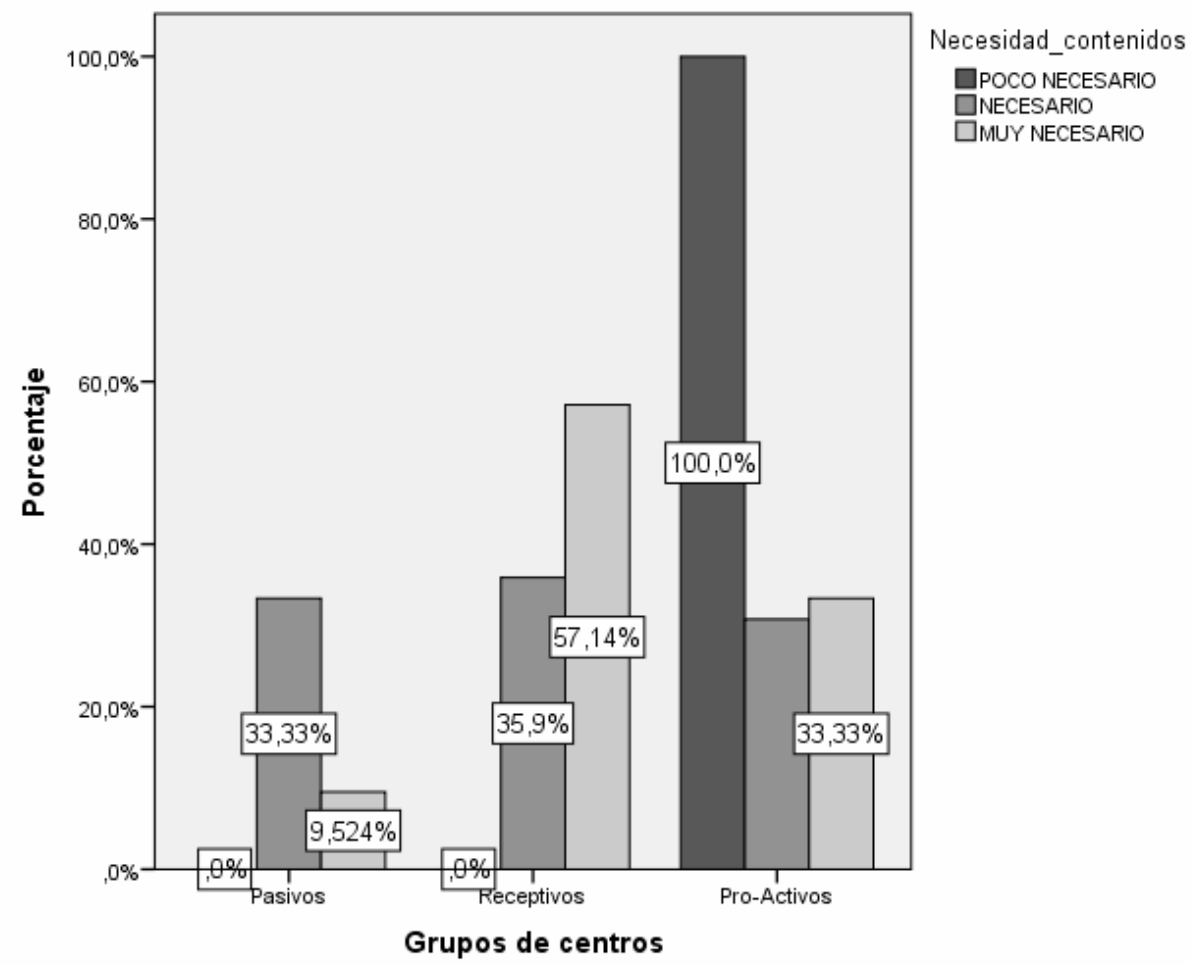

Figura 4. Cruce de grupos con necesidad de enseñar ECG en horario lectivo Fuente: Elaboración propia.

El 100\% de los centros que consideran "poco necesario" enseñar los contenidos en horario lectivo pertenecen al grupo proactivo (también han puntuado alto la actividad extraescolar), los centros que consideran "necesario" se reparten un 33,33\% en el grupo pasivo, un $36,9 \%$ en el grupo receptivo y un $30,67 \%$ en el de proactivo. Por último, los centros que consideran "muy necesario" se distribuyen en un $9,52 \%$ en pasivos, un $57,14 \%$ en receptivos y un $33,33 \%$ en proactivos. 
Cuadro 2. Pruebas chi-cuadrado. Cruce: grupos y necesidad de la ECG en horario lectivo

\begin{tabular}{lccc}
\hline & VAlOR & GL & $\begin{array}{c}\text { SIG. ASINTótICA } \\
\text { (2 CARAS) }\end{array}$ \\
\hline Chi-cuadrado de Pearson & $11,656^{\text {a }}$ & 4 & 0,020 \\
Razón de verosimilitud & 12,167 & 4 & 0,016 \\
Asociación lineal por lineal & 0,555 & 1 & 0,456 \\
N de casos válidos & 83 & & \\
\hline Nota: a 3 casillas (33,3\%) han esperado un recuento menor que 5. El recuento mínimo esperado es \\
O,41.
\end{tabular}

Si se atiende a con qué agentes se coordinan para realizar acciones de ECG en cada uno de los grupos, se observan diferencias significativas con todos los agentes (pruebas de Chicuadrado de Pearson, Sig. Asintótica-2 caras, con valores menores de 0,05, entre 0,00 y $0,02)$.

Respecto a la coordinación con los agentes externos, tal y como se observa en la Figura 5, los pasivos no señalan prácticamente coordinación en el centro ni con otros agentes. Los centros receptivos, la mayoría de ellos, realizan acciones coordinadas de forma preferente con agentes externos: ONG (65,79\%), asociación local (66,67\%), alguna institución $(60,53 \%)$ y con toda la comunidad educativa $(54,17 \%)$. En segundo lugar, señalan acciones coordinadas entre el claustro y otros docentes.

En el caso de los centros proactivos la relación se invierte con los diferentes agentes, ya que el claustro y el equipo docente tienen un papel clave.

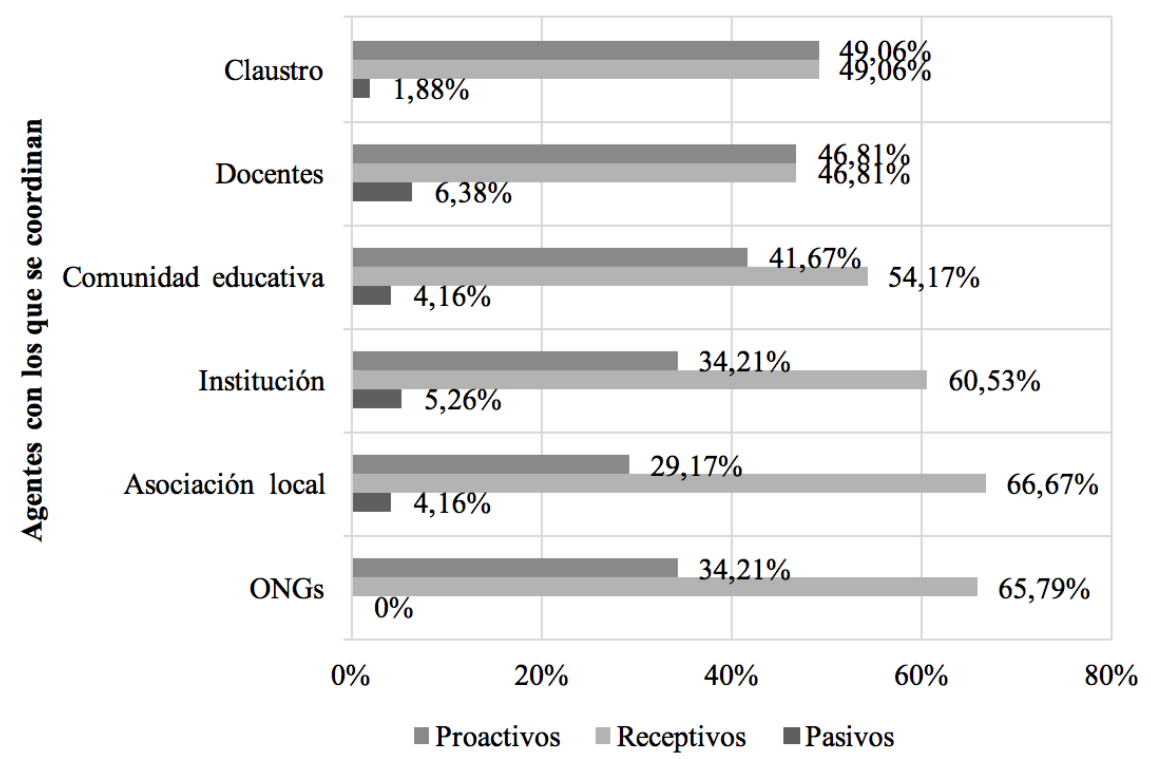

Figura 5. Cruce de grupos por agentes con los que se coordinan en acciones de ECG

Fuente: Elaboración propia.

En el caso de acciones coordinadas dentro del ámbito del centro: con otros docentes (46,81\%), con el claustro (49,06\%), el porcentaje de acciones del grupo receptivo coincide con el del grupo proactivo, también la tendencia es similar en cuanto a la coordinación con la comunidad educativa $(54,17 \%$ y $41,17 \%$ respectivamente). El grupo pasivo muestra 
índices de coordinación muy bajos, en ningún caso superior al 7\%. El contraste entre los grupos y el tipo de acciones educativas desarrolladas de ECG, muestra diferencias significativas con todos los tipos de acciones (pruebas de Chi-cuadrado de Pearson, Sig. Asintótica -2 caras, con valores entre 0,01 y 0,05).

La figura 6 muestra las diferencias entre los tipos de acciones desarrolladas por cada grupo. En el grupo receptivo, en contraste con los otros grupos, existe un mayor porcentaje, de los siguientes tipos de actividad: programación de un área/materia desde un enfoque de ECG $(76,47 \%)$, actividades a través de un proyecto transversal que implique a todo el centro $(50,00 \%)$, actividades puntuales de sensibilización $(48,61 \%)$ y actividades ligadas a tutorías $(48,57 \%)$.

En el grupo proactivo los tipos de actividad preferentes con respecto a los otros grupos son las actividades en el marco de un proyecto a corto-medio plazo $(60,00 \%)$ y actividades fuera del horario lectivo (69,23\%). En el grupo pasivo ninguna de ellas supera el 16\% de los centros encuestados, aun así, se observa que las más destacadas son puntuales y ligados al currículum: tutoría o materias.

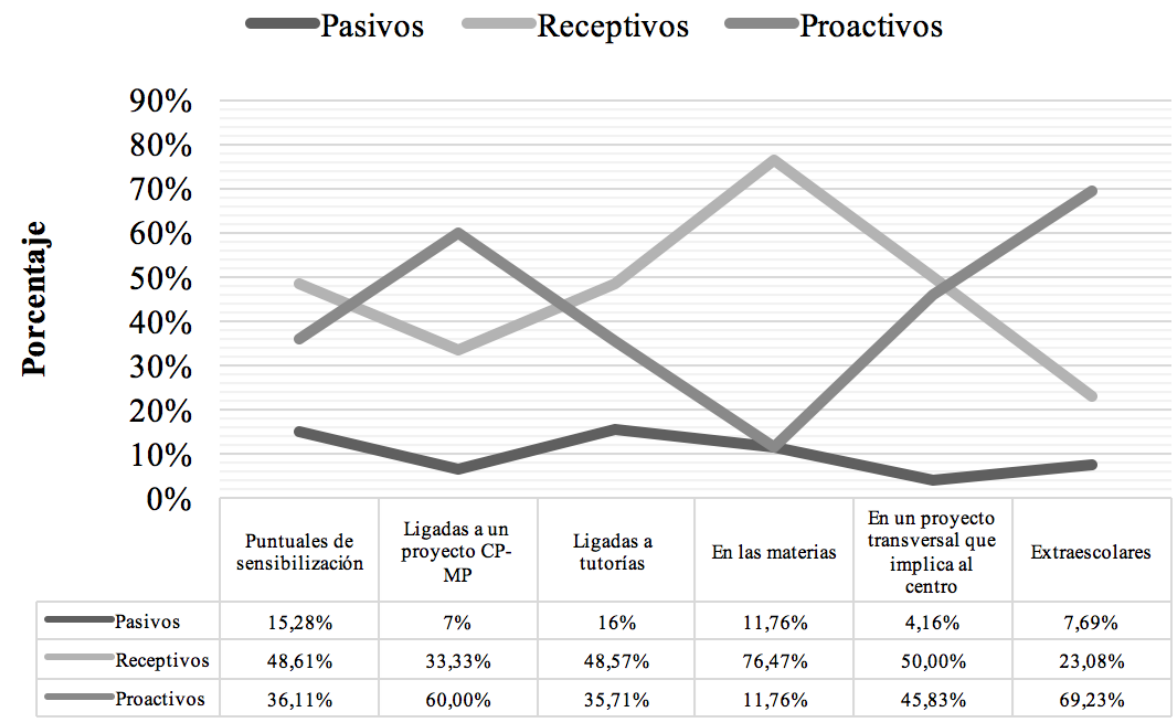

Tipo de acciones

Figura 6. Cruce de grupos por tipos de acciones donde se desarrolla ECG Fuente: Elaboración propia.

El contraste entre grupos, sobre qué agentes educativos toman la iniciativa para desarrollar acciones de ECG, muestra diferencias significativas respecto a si la iniciativa es del profesorado, del equipo directivo, del alumnado y de agentes del entorno (pruebas de chi-cuadrado de Pearson, Sig. Asintótica -2 caras, con valores entre 0,00 y 0,44). Cuando la iniciativa es de la familia $(0,98)$ o de agentes externos expertos en ECG $(0,27)$, no resulta significativa la diferencia entre grupos.

En el grupo receptivo las iniciativas surgen, en su mayoría, de la familia (71,30\%). El alumnado es el segundo colectivo en proponer iniciativas (60,71\%), seguido de agentes del entorno $(56,76 \%)$ y de agentes externos expertos en ECG $(54,50 \%)$. El equipo directivo $(52,40 \%)$ y el profesorado $(48,57 \%)$ presentan iniciativas en un menor porcentaje. Los 
porcentajes en el grupo proactivo oscilan entre el $21,3 \%$ y $37,84 \%$. De este modo, el origen de las iniciativas parte de todos los agentes en un porcentaje similar.

El grupo pasivo muestra índices de iniciativa muy bajos en todos los agentes, en ningún caso superior al $15,1 \%$. En la figura 7 se puede observar cómo los tres grupos mantienen un rango similar de porcentajes en relación a esta variable. No obstante, en los centros receptivos hay más iniciativa por parte de todos los agentes que en los centros proactivos.

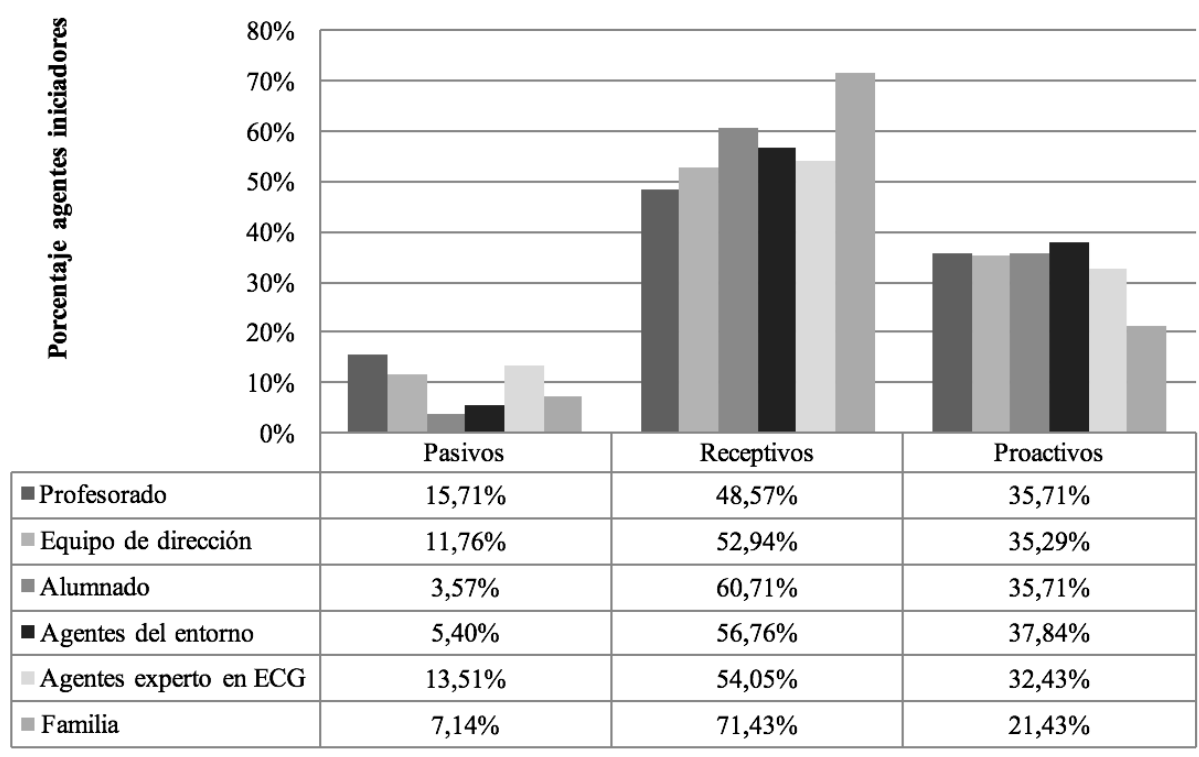

Figura 7. Cruce de grupos en relación con qué agente inicia acciones de ECG Fuente: Elaboración propia.

El contraste sobre cuál es la fuente de los materiales empleados en ECG muestra diferencias significativas, entre los grupos, cuando proceden de ONGS u otros agentes específicos, de editoriales o buscados en otras fuentes (pruebas de chi-cuadrado de Pearson, Sig. Asintótica -2 caras, con valores entre 0,01 y 0,04). Cuando los elaboran la Administración $(0,08)$, varios docentes $(0,36)$ o elaborados de forma individual $(0,10)$, no es significativa la diferencia.

En el grupo receptivo, se puede observar que hay un mayor porcentaje en el siguiente orden, cuando la procedencia es: ONGS u otros agentes específicos $(53,06 \%)$, buscados en otras fuentes $(53,30 \%)$, varios docentes $(47,06 \%)$ y elaborados de forma individual $(46,10 \%)$. Cuando la procedencia es de la Administración, el porcentaje más alto (50\%) coincide en el grupo receptivo y proactivo. En el grupo proactivo, únicamente en la procedencia de editoriales tienen mayor porcentaje (64,29\%).

Teniendo en cuenta todos estos resultados, se puede observar que la mayoría de los centros educativos resultan ser receptivos $(42,80 \%)$ indistintamente en Educación Primaria que en Educación Secundaria. Este hecho refleja un enfoque orientado a dar respuesta a un modelo educativo de centro (educación integral, desarrollo de valores, identidad...) pero con un desarrollo limitado de acciones de ECG. En estos centros hay menos coordinación y menor número de iniciativas. Además, las acciones educativas se desarrollan en un área o materia concreta, y en menor medida, de forma transversal, puntual o en tutoría. Por tanto, no se plantea en los centros educativos un proyecto a largo plazo, ni van más allá del horario lectivo. Sus fuentes principales para la elaboración de 
materiales relacionados con la ECG son las Organizaciones no Gubernamentales, ya que en su mayoría los docentes de estos centros desarrollan acciones de ECG a partir de propuestas ya establecidas por otros agentes. En relación con los aspectos de mejora, destacan la sistematización y desarrollo de proyectos orientados a acciones de ECG a largo plazo con una mayor coordinación.

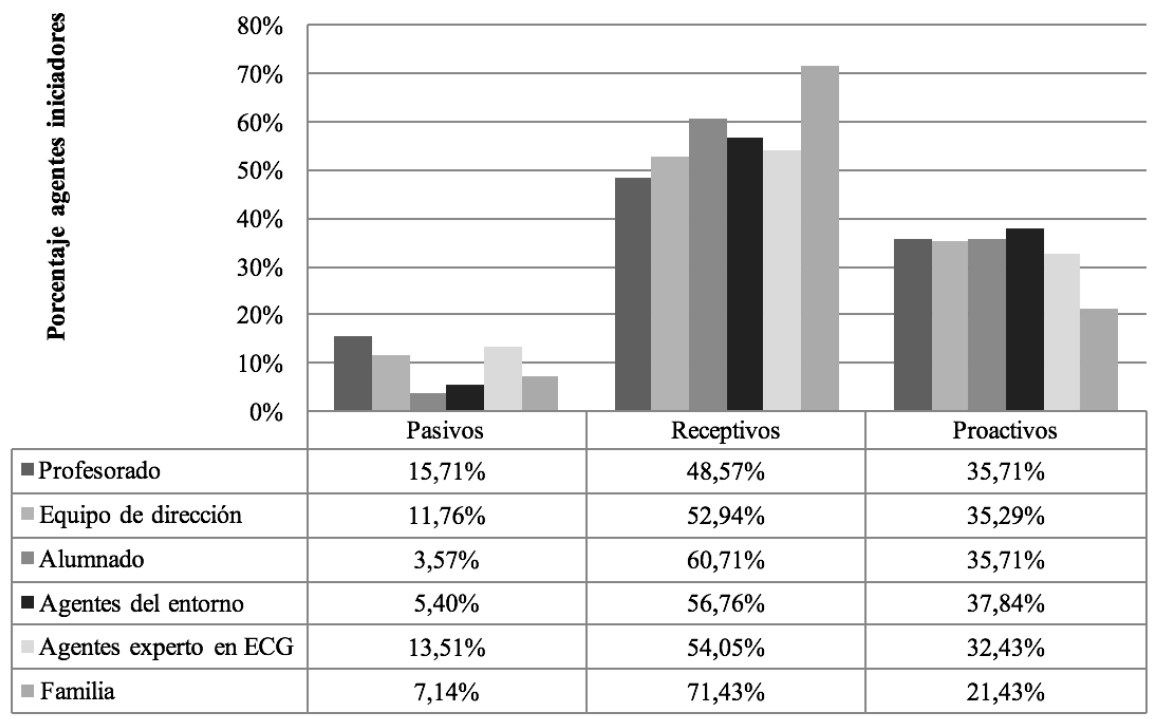

Figura 8. Cruce de grupos con la fuente de los materiales utilizados en ECG Fuente: Elaboración propia.

Respecto a los centros proactivos (35,10\%), hay que destacar que en Educación Primaria hay casi el doble que en Educación Secundaria. En ellos se realizan acciones de ECG de forma coordinada, preferentemente con otros docentes y con todo el claustro. Además de estar orientados al desarrollo del modelo de centro, se incide en la convivencia y en la atención a la diversidad; todo ello buscando que sean proyectos a corto-medio plazo y también fuera del horario lectivo. Las iniciativas pueden surgir de cualquiera de los agentes y hay una preferencia por buscar materiales en editoriales. En este grupo las propuestas de mejora inciden en mayor medida en la Administración: mayor dotación de recursos y medios, estabilidad de plantillas y en la legislación, y necesidad de apoyos institucionales.

\section{Discusión}

La configuración de los centros educativos en pasivos, receptivos y proactivos permite reflexionar sobre su actitud respecto al desarrollo e implementación de acciones de ECG. Los tipos de centros en conexión con las categorías resultantes (motivación y aspectos de mejora a futuro), muestran que el desarrollo de estas acciones se considera un potente instrumento para poder transformar el entorno y la sociedad desde las aulas (Bowers, 2004; Wang y Hoffman, 2016). A su vez, los centros educativos son considerados como escenarios privilegiados para poder desarrollar acciones de ECG (Digón et al., 2017). Para abordar este reto, hay que tener en cuenta la diversidad de creencias sobre los objetivos de la ECG (Reichert y Torney-Purta, 2018), que tienen relación con las características de 
cada docente inmerso en un contexto específico (Knowles, 2017; Thornberg y O'guz, 2016).

Para que las acciones de ECG adquieran un carácter integral y global se considera imprescindible articularlas en proyectos de centro en los que participe y colabore toda la comunidad educativa (Boni, 2017), en consonancia con una educación para la ECG centrada en el diálogo y basada en redes (Boni-Aristizábal, Hofmann-Pinilla y Sow-Paino, 2012). No es suficiente ofrecer contenidos, sino que es fundamental formar a los ciudadanos en valores, en un espíritu crítico, en responsabilidad con y para una sociedad solidaria y justa (Pineda-Alfonso, 2015; Pugliese, 2015; Santos, 2000).

Teniendo en cuenta los resultados de este estudio, deberemos considerar la predominancia del estilo receptivo en los centros educativos tanto en Educación Primaria como en Secundaria; así como la importancia de facilitar el cambio en los centros pasivos y aumentar la proactividad tanto en Primaria como en Secundaria.

En los centros proactivos, subyace al proceso de planificación, un compromiso con la labor docente, una reflexión y cuestionamiento de la práctica educativa (Bolívar, 1997; Gimeno y Pérez, 1992). Tal y como plantea Brown (2018), estos centros, deberían trabajar de manera activa, planificada y sistemática, desarrollando proyectos a largo plazo de forma coordinada y, alejándose así, de acciones puntuales y poco significativas. Sería interesante que los centros se orientaran en todas estas direcciones (implicando y coordinándose con los diferentes agentes, generando que las iniciativas puedan venir de cualquier agente y ampliando las fuentes de información) para que la ECG sea una experiencia real y mejore la realidad educativa y social desarrollando una metodología más participativa y de acción (Amiginho, 2011; Willemse et al., 2015).

En este sentido, el profesorado se orienta hacia una escuela rural que hace las veces de "laboratorio de renovación escolar" (Sepúlveda Ruiz y Gallardo Gil, 2011, p. 8). Los docentes apuestan por un modelo educativo de centro orientado al desarrollo de valores y actitudes; bien porque se prima la formación integral, o porque forma parte del currículo (Banks, 2017), o como una apuesta del proyecto educativo, o bien porque tienen como prioridad la educación en valores (Estellés y Romero, 2016; Goren y Yemini, 2017; Reichert y Torney-Purta, 2018). También puede ser debido, tal y como señalan Sepúlveda Ruiz y Gallardo Gil (2011) o Boix (2011), a la propia de idiosincrasia de la escuela rural, configurada en un contexto con necesidades y peculiaridades propias (Del Molino, 2016)

En el desarrollo de acciones de ECG en los centros educativos, se presenta el desafío de cómo diseñar acciones y estimular la generación de nuevos proyectos e ideas que fomenten la participación de las familias y las entidades del entorno ampliando las posibilidades de coordinación con todos los agentes (Garreta, 2013). A través de estas acciones, las escuelas muestran compromiso por el cambio y una cultura de colaboración y de trabajo conjunto por un fin común en el que los valores de solidaridad, autonomía, diálogo, colaboración y pensamiento crítico articulan un cambio educativo (Bolívar, 2013). El aislamiento de los centros educativos, tal y como señalan en su estudio Martínez y otros (2017), requiere de mayor sensibilización y capacitación de los docentes (aspecto señalado por el grupo receptivo) para dejar de ser "islas" en el marco educativo. Este aspecto, toma aun mayor relevancia en el caso de los docentes de escuelas rurales, que demandan, además, una formación más específica sobre el desarrollo de los procesos de enseñanza-aprendizaje en el marco rural (Boix, 2011). 
En el ámbito de la Administración, se plantea la importancia de que exista una mayor coordinación y coherencia con y entre las Administraciones (tal y como pone de relevancia el grupo proactivo). Se resalta la falta de estabilidad de la plantilla en el ámbito rural como un aspecto que impide que los centros educativos sean más proactivos, y puedan impulsar y desarrollar proyectos de ECG (Banks, 2017; Brown, 2018). En este sentido, los claustros son considerados "estructuras de funcionamiento, organizativas y de oportunidad en la práctica de la ciudadanía global” (Boni-Aristizabal, Hofmann-Pinilla y Sow-Paino, 2012, p.77).

Por ello, un aspecto fundamental de las estrategias planteadas es la institucionalización de la ECG a través de la normativa educativa tanto curricular como organizativa de centro, que permita desarrollar proyectos integrales y sistemáticos (Brown, 2018; GómezQuintero et al., 2017). De este modo, los centros educativos, que ya son proactivos, se verán más apoyados y los centros receptivos podrán ampliar su trabajo y desarrollo de la ECG y los pasivos tendrán ante sí la necesidad de un cambio, así como de empezar a plantearse la realización de estos proyectos y acciones.

\section{Conclusiones}

En definitiva, para que la ECG sea un hecho en el ámbito educativo formal, y más concretamente en el ámbito rural, es necesario que se planifique y coordine desde una actitud de compromiso y responsabilidad social de toda la comunidad educativa, si bien, la dispersión y limitación de estas prácticas puede ser un aspecto muy común en nuestro sistema educativo. A su vez, estas experiencias deben ser analizadas y significadas como motor de una corriente pedagógica para una Ciudadanía Global.

Consideramos que el potencial didáctico y social del territorio rural se presenta como un escenario clave para trabajar y desarrollar los principios de la ECG. Las áreas rurales y más concretamente sus escuelas pueden establecerse como marcos de referencia para el desarrollo de un compromiso efectivo con la comunidad que permita al alumnado interactuar en una sociedad globalmente interdependiente.

No obstante, el desconocimiento, en muchos de los casos, del sentido y significado de la ECG hace más complicado conocer qué agentes desarrollan este concepto en los entornos educativos y, en su caso, cómo lo hacen. Por ello, sería de gran interés científico poder profundizar en el estudio de los tres tipos de centros derivados de este estudio (proactivos, receptivos y pasivos), desde una perspectiva constructivista (Rodríguez, Gil y García, 1996). La investigación se abordaría desde los escenarios educativos no solo para describirlos, sino también para conocer cómo, por qué y para qué se desarrollan las distintas iniciativas y prácticas de ECG.

A partir de este estudio se nos plantean nuevos interrogantes como ¿en qué sentido los entornos rurales permiten un mejor y mayor desarrollo de la ECG que los entornos urbanos?, ¿Cómo se puede fomentar una mayor proactividad hacia la ECG en los docentes de los centros rurales?, ¿Cuáles son las claves para que los proyectos de los centros sean proyectos trasversales, comunitarios, a largo plazo y con incidencia local y global?

Existe poca investigación sobre el desarrollo de la ECG en el ámbito rural. Por ello, sería necesario diseñar y desarrollar estudios rigurosos que permitan construir conocimiento sobre este tema tan específico y de tanta relevancia para que exista cada vez más compromiso activo en los docentes y en la comunidad rural con el bien común. 


\section{Agradecimientos}

A todos los agentes educativos implicados en los centros educativos de la provincia de Zaragoza que han participado y colaborado en esta investigación.

\section{Financiación}

Este estudio forma parte del Diagnóstico de la Educación para el Desarrollo y la Ciudadanía Global en la Provincia de Zaragoza (Fase 1), realizado a través de un Convenio entre la Diputación Provincial de Zaragoza, la Cátedra de Cooperación para el Desarrollo de la Universidad de Zaragoza y la Federación Aragonesa de Solidaridad.

\section{Referencias}

Amiguinho, A. (2011). La escuela en el medio rural: Educación y desarrollo local. Profesorado. Revista de Currículum y Formación de Profesorado, 15(2), 25-37.

Banks, J. A. (2017). Diversity, group identity, and citizenship education in a global age. Journal of Education, 194(3), 1-12. https://doi.org/10.1177/002205741419400302

Blasco-Serrano, A. C., Coma, T. y Dieste, B. (Coords.). (2018). II Fase: Diagnóstico de la educación para el desarrollo y la ciudadanía global en la Provincia de Zaragoza. Recuperado de http://catedradecooperacion.unizar.es/sites/default/files/ 1.\%20INFORME\%20FINAL\% 20Fase\%202.pdf

Bolívar, A. (2010). La lógica del compromiso del profesorado y la responsabilidad del centro escolar. Una revisión actual. REICE. Revista Iberoamericana sobre Calidad, Eficacia y Cambio en Educación, 8(2), 11-33.

Bolívar, A. (2013). La lógica del compromiso del profesorado y la responsabilidad del centro escolar: una nueva mirada. REICE. Revista Iberoamericana sobre Calidad, Eficacia y Cambio en Educación, 11(2), 60-86.

Boni, A. (2011). Educación para la ciudadanía global. Significados y espacios para un cosmopolitismo transformador. Revista Española de Educación Comparada, 17, 65-85.

Boni, A. (2017). From practice to policy: Reflections from the participatory process to design a development education strategy in Valencia (Spain). Policy \& Practice: A Development Education Review, 25, 109-211.

Boni-Aristizabal, A., Hofmann-Pinilla, A. y Sow-Paino, J. (2012). Educando para la ciudadanía global. Una experiencia de investigación cooperativa entre docentes y profesionales de las ONGD. Estudios sobre Educación, 23, 63-81.

Boix, R. (2011). ¿Qué queda de la escuela rural? Algunas reflexiones sobre la realidad pedagógica del aula multigrado. Profesorado. Revista de Currículum y Formación del Profesorado 15(2), 1323 .

Bowers, C. A. (2004). Revitalizing the commons or an individualized approach to planetary citizenship: The choice before us. Educational Studies, 36(1), 45-58. https://doi.org/10.1207/s15326993es3601_5

Briones, G. (1996). Metodología de la investigación. La encuesta social. Módulos de investigación social. Bogotá: Icfes. 
Braun, V. y Clarke, V. (2006). Using thematic analysis in psychology. Qualitative Research in Psychology, 3(2), 77-101. https://doi.org/10.1191/1478088706qp063oa

Brown, R. (2018). Knowledge, education, and cultural change. Londres: Routledge. https://doi.org/10.4324/9781351018142

Cabrera, F. A. (2007). Elaboración y evaluación de programas de educación para la ciudadanía. Bordón. Revista de Pedagogía, 59(2), 375-400.

Celorio, J. J. (2013). Educación para el desarrollo: Educar en y para la cooperación y la solidaridad. Contextos Educativos. Revista de Educación, 3, 17-44.

Contreras, D. y Aceituno, D. (2017). Learning to teach citizenship competencies through a schooluniversity collaborative action research project. Procedia-Social and Behavioral Sciences, 237, 90-95. https://doi.org/10.1016/j.sbspro.2017.02.035

Del Molino, S. (2016). La España vacía. Viaje por un país que nunca fue. Madrid: Turner Libros.

Delors, J., Al Mufti, I., Amagi, I., Carneiro, R., Chiung, F., Geremek, B. y Nanzhao, Z. (1996). La educación encierra un tesoro. Informe a la UNESCO de la comisión internacional sobre la educación para el siglo XXI. París: UNESCO.

Digón, P., Méndez, R.M., De Palma, R. y Longueira, S. (2017). A place for development education in the current Spanish and English curricula: Finding possibilities for practice. International Journal of Development Education and Global Learning, 9(2), 97-114. https://doi.org/10.18546/IJDEGL.09.2.04

Engel, L. C., Fundalinski, J. y Cannon, T. (2016). Educación para la ciudadanía global a nivel local: un análisis comparado de cuatro distritos urbanos estadounidenses. Revista Española de Educación Comparada, 28, 23-51. https://doi.org/10.5944/reec.28.2016.17095

Estellés, M. y Romero, J. (2016). Teacher education for citizenship in a globalized world: A Case study in Spain. Journal of International Social Studies, 6(2), 21-33.

García, F. y De Alba, N. (2009). Educar para la participación ciudadana. Análisis de las dificultades del profesorado a partir de la experiencia del programa Parlamento Joven. Bolonia: Pàtron Editore.

Garreta, J. (2013). La participación de las familias en la escuela: Una cuestión pendiente. Documentación Social. Revista de Estudios Sociales y Sociología Aplicada, 171, 101-124.

Gimeno Sacristán, J. (2001). Educar y convivir en la cultura global. Madrid: Morata.

Gómez-Quintero, J. D., Moreno, J. R., Jacotte, C., Almaguer, P., Cabezudo, E., Tapiador, R. y Ramos, R. (2017). Diagnóstico de la educación para el desarrollo y la ciudadanía global. Recuperado de http://www.dpz.es/areas/bienestar-social-y-desarrollo/ficheros/ diagnostico-educacion-para-el-desarrollo-y-la-ciudadania-global/view

Gómez, F. J., Domenech, E. y Carracedo, M. (2012). Propuesta metodológica para hacer un diagnóstico de la educación para el desarrollo. Revista Electrónica Interuniversitaria de Formación del Profesorado, 15(2), 37-46.

Goren, H., y Yemini, M. (2017). Global citizenship education redefined-A systematic review of empirical studies on global citizenship education. International Journal of Educational Research, 82, 170-183. https://doi.org/10.1016/j.ijer.2017.02.004

Guo, L. (2014). Preparing teachers to educate for 21 st century global citizenship: envisioning and enacting. Journal of Global Citizenship \& Equity Education, 4(1), 75-98.

Kikuchi, T. (2003). Global citizenship education through study abroad programs with service learning experiences. Bulletin of Nagoya University of Foreign Studies, 2, 73-101. 
Kopish, M. A. (2017). Global Citizenship education and the development of globally competent teacher candidates. Journal of International Social Studies, 7(2), 20-59.

Knowles, R. T. (2017). Teaching who you are: Connecting teachers' civic education ideology to instructional strategies. Theory y Research in Social Education, 191(1), 21-42. https://doi.org/10.1080/00933104.2017.1356776

Maiztegui, C. (2007). La participación como una opción transformadora en los procesos de educación ciudadana. REICE. Revista Iberoamericana sobre Calidad, Eficacia y Cambio en Educación, 5(4), 144-160.

Martínez, I., Sánchez, García, B. y González, D. (2017). What do the teaching staff in three secondary schools with ISO 9001/2008 certification think about the quality of diversity improvement plans? Procedia-Social and Behavioral Sciences, 237, 237-241. https://doi.org/10.1016/j.sbspro.2017.02.069

OCUVa. (2017). Observatorio de cooperación internacional para el desarrollo de la Universidad de Valladolid. Valladolid: DPV/UV

Peña, M. L., Delgado, L. M. y Valverde, Y. (2014). Educación para una ciudadanía global. Fedumar Pedagogía y Educación, 1(1), 7-24

Pineda-Alfonso, J. A. (2015). Educar para la ciudadanía trabajando con temas controvertidos en Educación Secundaria Obligatoria. Revista de Investigación Educativa, 33(2), 353-367. https://doi.org/10.6018/rie.33.2.208441

Pigozzi, M. J. (2006). A UNESCO view of global citizenship education. Educational Review, 58(1), 1-4. https://doi.org/10.1080/00131910500352473

Pugliese, G. D. (2015). Preparing students for citizenship in a global society: A case study. Ann Arbor, CO: Southern Connecticut State University:

Reichert, F. y Torney-Purta, J. (2018). A cross-national comparison of teachers' beliefs about the aims of civic education in 12 countries: A person-centered analysis. Teaching and Teacher Education, 77, 112-115. https://doi.org/10.1016/j.tate.2018.09.005

Rodríguez, G., Gil, J. y García, E. (1996). Metodología de la investigación cualitativa. Madrid: Aljibe.

Rubio-Hurtado, M. J. y Vilà-Baños, R. (2017). El análisis de conglomerados bietápico o en dos fases con SPSS. REIRE. Revista d'Innovació $i$ Recerca en Educació, 1O(1), 118-126.

Rumayor, L. R. y de las Heras, A. M. (2012). Una escuela rural en transformación. De una ciudadanía local a una ciudadanía global. Profesorado. Revista de Currículum y Formación de Profesorado, 16(1), 325-343.

Sabirón, F. (2006). Métodos de investigación etnográfica en ciencias sociales. Zaragoza: Mira Ediciones.

Santos, M. A. (2000). La escuela que aprende. Madrid: Morata.

Sepúlveda Ruiz, M. y Gallardo Gil, M. (2011). La escuela rural en la sociedad globalizada: nuevos caminos para una realidad silenciada. Profesorado. Revista de Currículum y Formación del Profesorado, 15(2), 141-153.

Soriano, J. y Monsalve, V. (2004). Validación del cuestionario de afrontamiento al dolor crónico reducido (CAD-R). Revista de la Sociedad Española del Dolor, 11 (7), 27-34.

Torres, J. (2011). Currículum, justicia e inclusión. En J. Gimeno Sacristán (Ed.), Saberes e incertidumbres sobre el currículum (pp. 84-102). Madrid: Morata.

Thornberg, R. y O'guz, E. (2016). Moral and citizenship educational goals in values education: A cross-cultural study of Swedish and Turkish student teachers' preferences. Teaching and Teacher Education, 55, 110-121. https://doi.org/10.1016/j.tate.2016.01.002 
UNESCO. (2015a). Declaración de Incheon. Educación 2030. Recuperado de http://unesdoc.unesco.org/images/0023/002338/233813M.pdf

UNESCO. (2015b). Declaración de Incheon y marco de acción para la realización del objetivo de desarrollo sostenible 4. Educación 2030. Recuperado de http://unesdoc.unesco.org/images/0024/002456/245656s.pdf

Vigo, B. y Dieste, B. (2018). Building virtual interaction spaces between family and school. Ethnography and Education, 14(2), 206-222. https://doi.org/10.1080/17457823.2018.1431950

Vigo, B. y Soriano, J. (2014). Teaching practices and teachers' perceptions of group creative practices in inclusive rural schools. Ethnography and Education, 9(3), 253-269. https://doi.org/10.1080/17457823.2014.881721

Vigo, B., Dieste, B. y Thurston, A. (2016). Aportaciones de un estudio etnográfico sobre la participación de las familias a la formación crítica del profesorado en una escuela inclusiva. Revista Electrónica Interuniversitaria de Formación del Profesorado, 19(1), 1-14. https://doi.org/10.6018/reifop.19.3.267221

Wang, C. y Hoffman, D. M. (2016). Are we the world? A critical reflection on selfhood and global citizenship education. Education Policy Analysis Archives, 24(56), 1-22. https://doi.org/10.14507/epaa.24.2152

Willemse, T. M., Ten Dam, G., Geijsel, F., Van Wessum, L. y Volman, M. (2015). Fostering teachers' professional development for citizenship education. Teaching and Teacher Education, 49, 118-127. https://doi.org/10.1016/j.tate.2015.03.008

\section{Breve CV de las autoras}

\section{Ana Cristina Blasco-Serrano}

Doctora en Educación por la Universidad de Zaragoza. Licenciada en Pedagogía por la Universidad Rovira i Virgili, desempeña su labor docente como Profesora asociada en el Dpto. de Ciencias de la Educación de la Universidad de Zaragoza. Miembro del Grupo de Investigación Aplicada en Etnografía de la Educación (Etnoedu). Miembro del Consejo Asesor de la Cátedra de Cooperación de la Universidad de Zaragoza. Sus líneas de investigación y de docencia se centran en la Educación para la Ciudadanía Global y su inclusión en el currículo educativo, la orientación educativa desde una perspectiva inclusiva y la inclusión de la Tecnología para el Aprendizaje y la Comunicación en los procesos de enseñanza-aprendizaje. ORCID ID: https://orcid.org/OOOO-OOO2-42942982. Email: anablas@unizar.es

\section{Belén Dieste}

Doctora en Educación (Universidad de Zaragoza). Licenciada en Filosofía y Ciencias de la Educación (UNED). Diplomada en Magisterio Audición y Lenguaje (Universidad de Zaragoza). Título de Especialista Universitario en Educación Infantil (UNED). Profesora Asociada (Departamento de Ciencias de la Educación. Universidad de Zaragoza) Miembro del Grupo de Investigación EDI "Educación y Diversidad". Miembro del Consejo Asesor de la Cátedra de Cooperación de la Universidad de Zaragoza. Líneas de investigación: Escuela Inclusiva, Participación de las Familias en la Escuela, Aprendizaje y Servicio, Formación del Profesorado y Educación para la Ciudadanía Global. ORCID ID: https://orcid.org/OOOO-0002-7262-896X. Email: bdieste@unizar.es 


\section{Teresa Coma}

Doctora en Psicología y Aprendizaje (Universidad de Zaragoza). Licenciada en Pedagogía Social (Universitat Autónoma de Barcelona), Diplomada en Magisterio Educación Especial (Universidad de Zaragoza) y Educadora Social. Máster Intervención Psicológica con DBM. Profesora Asociada (Departamento de Psicología y Sociología. Universidad de Zaragoza). Grupo de Investigación en Interfaces Avanzadas /AffectiveLab y del Grupo internacional TEIAS. Miembro del Consejo Asesor de la Cátedra de Cooperación de la Universidad de Zaragoza. Líneas de investigación: empatía, orientación, participación y trabajo en equipo, competencias interpersonales, gestión del desempeño, inclusión y Educación para la Ciudadanía Global. ORCID ID: https://orcid.org/0000-0002-74398459. Email: tcoma@unizar.es 